\title{
Physical Fitness Differences Between Prepubescent Boys and Girls
}

\author{
Carlos C. Marta, ${ }^{1,2}$ Daniel A. Marinho, $, 2,3$ Tiago M. Barbosa, ${ }^{2,4}$ Mikel Izquierdo, ${ }^{5}$ and \\ Mário C. Marques ${ }^{2,3}$ \\ ${ }^{1}$ Department of Sport Sciences, Polytechnic Institute of Guarda, Education, Communication, and Sport School, IPG, Guarda, \\ Portugal; ${ }^{2}$ Research Center in Sports, Health and Human Development, CIDESD, Portugal; ${ }^{3}$ Department of Sport Sciences, \\ University of Beira Interior, Covilhã, Portugal; ${ }^{4}$ Department of Sport Sciences, Polytechnic Institute of Bragança, Bragança, \\ Portugal; and ${ }^{5}$ Department of Health Sciences, Public University of Navarre, Navarre, Spain
}

\begin{abstract}
Marta, CC, Marinho, DA, Barbosa, TM, Izquierdo, M, and Marques, MC. Physical fitness differences between prepubescent boys and girls. J Strength Cond Res 26(7): 1756-1766, 2012-The purpose of this study was to analyze in which physical capabilities boys and girls are closer or distant. An additional objective was to find which of the body fat, physical activity, and somatotype factors have a greater effect on prepubescent children's physical fitness. This was a cross-sectional study involving 312 children (10.8 \pm 0.4 years). The physical fitness assessment employed sets of aerobic fitness, strength, flexibility, speed, agility, and balance. The boys presented higher values in all selected tests, except tests of balance and flexibility, in which girls scored better. Gender differences in the physical fitness were greatest in the explosive strength of upper $\left(p \leq 0.01, \eta_{p}^{2}=0.09\right)$ and lower limbs ( $\left.p \leq 0.01, \eta_{p}^{2}=0.08\right)$, although with a medium-size effect of gender, and smaller in the abdominal $\left(p>0.05, \eta_{p}^{2}=0.007\right)$ and upper limbs $(p>0.05$, $\left.\eta_{p}^{2}=0.003\right)$ muscular endurance, and trunk extensor strength and flexibility $\left(p>0.05, \eta_{p}^{2}=0.001\right)$. The endomorphic ( $p \leq$ $\left.0.01, \eta_{p}^{2}=0.26\right)$ in the girls, and the ectomorphic ( $p \leq 0.01$, $\left.\eta_{p}^{2}=0.31\right)$ and mesomorphic ( $\left.p \leq 0.01, \eta_{p}^{2} 0.26\right)$ in the boys, had the high-sized effect on the physical fitness. The physical activity in the girls, and the endomorphic and body fat in the boys, did not have a significant effect. These findings can help in the planning of activities that take into account the success and motivation of both boys and girls and thus increase levels of physical activity and physical fitness at school. However, in prepubescent children, one cannot
\end{abstract}

Address correspondence to Dr. Mikel Izquierdo, mikel.izquierdo@ gmail.com.

26(7)/1756-1766

Journal of Strength and Conditioning Research

(C) 2012 National Strength and Conditioning Association neglect the influence of genetic determinism, observed from the morphoconstitutional point of view.

KEY WoRDS gender difference, somatotype, motor performance, school

\section{INTRODUCTION}

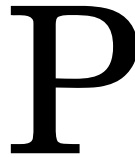
hysical fitness has been recognized as a key determinant in healthy lifestyles based increasingly on criteria referenced to general health and not merely to motor performance (30). It has positively been related, among others, with benefits to cardiovascular, total and abdominal adiposity, skeletal health, depression, anxiety, self-esteem, and higher academic performance $(19,30)$. However, many children and adolescents are only exposed to vigorous physical activity during schoolbased physical education classes (9). That way, schools seem to provide an excellent setting to enhance physical activity and physical fitness levels.

Unfortunately, it has been observed that there is an apparent avoidance of children of the physical education classes and regular physical activity practice at school (33). This decrease in the interest of children in physical education is partly because of the lack of planning that takes into account the interest, motivation, and success of children in the execution of the exercises, respecting the differences among students, including boys and girls (14). Physical education classes or extracurricular activities commonly include children of both sexes. This requires teachers to establish a match between the goals they want to achieve and the means and resources available, taking into account the various constraints that may arise, such as reduced practice time per session, number of weekly sessions, lack of material resources and facilities, high numbers of students and athletes by class, and variety of content to be taught, often involving the same activities for boys and girls alike.

In this respect, beyond the duty of the teacher to maximize the opportunities for practice, it is necessary to program the exercises in school to make them appealing and motivating for both boys and girls (29). The psychological saturation and

1756 Journal of Strength and Conditioning Research 
physiological enervation that may attend on attempts to match results close to those achieved by opposite-sex colleagues in the same exercise can be alleviated when there is knowledge about which exercises and the respective capabilities they deploy can be best performed concurrently by boys and girls. One of the major reasons why many children drop out of sport and physical activity is that they feel they do not have the necessary skills to be involved. Both girls and boys tend to be reluctant to participate if their perceived level of skill is low. The proof of this is that the female students reported more positive and adaptive perceptions in same-sex classes (22). Thus, the knowledge, not only of the physical capabilities in which boys and girls are higher but also the magnitude of the differences between them, can help teachers in the planning and organizing of activities at school, increasing the motivational levels in the exercises and thus, increasing levels of physical activity and physical fitness of their students. Also, at the level of assessment, this knowledge may add value in allowing the teacher to better analyze and understand the results obtained in general.

On the other hand, for a better understanding of gender differences in physical fitness, it will be important to understand the interaction of several factors referred in the literature with the motor performance, in both boys and girls. There is much evidence pointing to significant differences between boys and girls in motor performance $(8,15)$. These differences are largely because of gender differences in levels of habitual physical activity (37) and body fat $(8,20)$. Although body fat is associated negatively with several motor tasks, particularly those related to the propulsion and lift movements of the body (11), higher levels of physical activity represent a gain in motor performance in general (36). However, there is a relative paucity of reports focused on the differences in the physical fitness between prepubertal boys and girls regarding his or her somatotype. Most studies in this context refer to the influence of body mass index in the motor performance in youth, but somatotype has been found to be inherited to a greater extent than body mass index (32). The values of relative adiposity, relative muscle-skeletal magnitude, (robustness) or relative thinness of the subjects (23), allow performances to vary depending on the type of motor tasks (17). One cannot exceed the limits imposed by what is a manifestation of genetic determinism, observed from the morphoconstitutional point of view, and there is evidence that by prepuberty there already exists a fairly stable somatotype, pointing to 8 years as the age by which somatotype stability becomes manifest $(23,24)$. Hence, it seems relevant to examine, in this age group, the effect of the presence or absence of certain physical traits on the motor performance and compare it with the effect of body fat and physical activity, often referenced in the literature.

The aim of this investigation was to analyze in which physical capabilities boys and girls are closer or distant. An additional objective was to find which of the body fat, physical activity, and somatotype factor is more interactive with prepubescent children's physical fitness level. It was considered the hypothesis that there is a set of exercises and physical capabilities inherent to them, in which the performance of boys and girls is very similar or divergent. We also hypothesized that, despite the age group considered, somatotype already plays a determinant role on the performance of selected exercises, in both boys and girls.

\section{Methods}

\section{Experimental Approach to the Problem}

It has been observed that there is an apparent decrease in the interest of children in physical education partly because of the lack of planning that takes into account the success of children in the execution of the exercises respecting the differences among students, including boys and girls. The knowledge of the magnitude of the differences between boys and girls in physical fitness can help the planning of activities that promote the success and motivation of both boys and girls and thus improve levels of physical activity and physical fitness at school. On the other hand, the knowledge of the effect of the somatotype on the performance in the prepuberty (comparing it with the effect of body fat and physical activity, often referenced in the literature) may also allow a better understanding of gender differences.

Three hundred and twelve students were recruited from a Portuguese public basic school (from fifth and sixth grades) to perform this study. In Portugal, a physical education class has a set of 45 minutes and another of 90 minutes twice a week. Typical physical education classes include various activities, in which participate simultaneously boys and girls, with a clear pedagogical focus, but mainly for the purpose of promoting levels of physical activity and physical fitness of students. Usually, these classes start with jogging run during 10 minutes to general warm-up, proceed to joint mobilization and general stretches. After that, the class is divided into 2 or 3 proficiency level groups to start the main activities and sports of the class.

The required data were collected (from January 4 to February 25, 2011) from self-assessed of maturity level, anthropometric measurements, questionnaire about habitual physical activity, and application of a battery of previously selected tests. All anthropometric measurements were carried out before any physical performance test. All the participants were familiarized with physical fitness tests, and the measurements were performed after a 10 -minute warm-up period (7-minute running with an intensity sufficient to raise breath rate, 3 -minute stretching and jointspecific warm-up). All measurements were made by the same investigator, in the first periods in the morning, and the testing assessment procedures were always conducted in the same indoor sportive facility (with temperature between 15 and $18^{\circ} \mathrm{C}$ ). In the course of conducting testing, there was a constant concern to ensure the necessary security and maintenance of safe hydration levels and to encourage all the children to achieve the best results. Clear instructions about the importance of adequate nutrition for physical 
activity were also given. The following exclusion criteria were used: subjects with a chronic pediatric disease or with an orthopedic limitation. Subsequently, to minimize the effects of growth, only the subjects who were self-assessed in Tanner stages 1-2 were selected (12).

\section{Subjects}

The sample, cross-sectional in type, consisted of 312 prepubescent children (160 girls, 152 boys) all of whom volunteered for this study. The age, height, and weight of the whole sample were $10.8 \pm 0.4$ years, $1.45 \pm$ $0.08 \mathrm{~m}, 40.0 \pm 8.7 \mathrm{~kg}$, respectively (girls: $10.8 \pm 0.4$ years, $1.44 \pm 0.07 \mathrm{~m}, 38.9 \pm 8.5 \mathrm{~kg}$; boys: $10.8 \pm 0.4$ years, $1.45 \pm$ $0.09 \mathrm{~m}, 41.2 \pm 8.8 \mathrm{~kg})$. Both boys and girls were in Tanner stages 1-2 (girls: stage 1, 53.1\% and stage 2 , $46.9 \%$; boys: stage 1 , $81.6 \%$ and stage $2,18.4 \%)$. This study was approved by the institutional review boards of the University of Beira Interior (UBI) and Research Centre in Sports, Health and Human Development (CIDESD), Portugal. An informed consent was obtained from all of the children and their parents/ guardians before testing.

\section{Procedures}

Parameters of body fat, somatotype, level of physical activity, and physical fitness were evaluated for all the subjects participating in the study (Table 1).

Anthropometric Measurements. All anthropometric measurements were assessed according to international standards for anthropometric assessment (25). The participants were barefoot and wore only underwear. Body weight (kilograms) was measured to the nearest $0.1 \mathrm{~kg}$ using a standard digital floor scale (Seca, model 841, Germany). To evaluate body height (centimeters), a precision stadiometer with a range scale of $0.10 \mathrm{~cm}$ was used (Seca, model 214). For perimeter measurement, a circumference tape was used (Seca 200). The bicondyle femoral and humeral diameters were assessed (Campbell, 20, Ross Craft, Canada). The percentage body fat (\%FAT) from skinfold anthropometry was calculated following the method of Slaughter et al. (35). As such, triceps and subscapular skinfolds were determined by internationally recommended methods (25). The definition of morpho- logical typology (TYPE) used the method described by Heath-Carter (16), expressed quantitatively by a score of 3 components: endomorphy (ENDO), mesomorphy (MESO), and ectomorphy (ECTO). Maturity level based on Tanner stages was self-assessed (12). The students were asked to answer to an image with corresponding legend questionnaire, in an individual booth, without interference from their teachers or friends.

Physical Activity Assessment and Physical Fitness Tests. The habitual physical activity level was measured using the Baecke et al. (5) questionnaire expressed quantitatively by a score of 3 indexes: physical activity in school, sport during leisure time, and physical activity during leisure time excluding sport.

For the assessment of physical fitness, motor tests were chosen to include the assessment of aerobic capability $(20-\mathrm{m}$ multistage shuttle run), flexibility of the lower back and hamstrings (left and right sit-and-reach), trunk extensor strength and flexibility (trunk lift), speed (20-m sprint), agility and coordination (9.14-m shuttle run), general stability (flamingo balance), muscle strength and endurance (curl-ups and push-ups), explosive strength (standing broad jump and medicine-ball throw), maximum isometric strength (handgrip strength), and anaerobic muscular power

1758 Journal of Strength and Conditioning Research 
TABLE 2. Gender difference in physical performance: Adjusted means after MANCOVA.

\begin{tabular}{|c|c|c|c|c|c|c|}
\hline & \multirow[b]{2}{*}{ Gender } & \multirow[b]{2}{*}{ Mean } & \multirow[b]{2}{*}{$S E$} & \multicolumn{2}{|c|}{$95 \% \mathrm{Cl}$} & \multirow[b]{2}{*}{$p^{*}$} \\
\hline & & & & LB & UB & \\
\hline \multirow[t]{2}{*}{ 20-m Shuttle run (a.u.) } & Female & 25.38 & 1.05 & 23.32 & 27.44 & $0.001 \ddagger$ \\
\hline & Male & 31.05 & 1.19 & 28.71 & 33.39 & \\
\hline \multirow[t]{2}{*}{ 20-m Sprint (s) } & Female & 4.52 & 0.04 & 4.45 & 4.60 & $0.047 \dagger$ \\
\hline & Male & 4.40 & 0.04 & 4.31 & 4.48 & \\
\hline \multirow[t]{2}{*}{ 9.14-m Shuttle run (s) } & Female & 13.56 & 0.10 & 13.36 & 13.77 & $<0.001 \ddagger$ \\
\hline & Male & 12.89 & 0.12 & 12.65 & 13.12 & \\
\hline \multirow[t]{2}{*}{ Flamingo balance (faults) } & Female & 7.55 & 0.50 & 6.56 & 8.53 & $0.011 \dagger$ \\
\hline & Male & 9.70 & 0.57 & 8.58 & 10.82 & \\
\hline \multirow[t]{2}{*}{ Sit-and-reach R (cm) } & Female & 22.95 & 0.50 & 21.96 & 23.93 & $0.001 \ddagger$ \\
\hline & Male & 20.07 & 0.57 & 18.95 & 21.18 & \\
\hline \multirow[t]{2}{*}{ Sit-and-reach $L(\mathrm{~cm})$} & Female & 23.03 & 0.52 & 22.01 & 24.04 & $<0.001 \dagger$ \\
\hline & Male & 18.62 & 0.59 & 17.47 & 19.78 & \\
\hline \multirow[t]{2}{*}{ Trunk lift $(\mathrm{cm})$} & Female & 23.75 & 0.44 & 22.87 & 24.62 & 0.583 \\
\hline & Male & 23.34 & 0.50 & 22.35 & 24.33 & \\
\hline \multirow[t]{2}{*}{ Curl-ups (a.u.) } & Female & 26.61 & 1.50 & 23.67 & 29.55 & 0.145 \\
\hline & Male & 30.27 & 1.70 & 26.93 & 33.62 & \\
\hline \multirow[t]{2}{*}{ Push-ups (a.u.) } & Female & 9.95 & 0.61 & 8.75 & 11.15 & 0.362 \\
\hline & Male & 10.89 & 0.70 & 9.52 & 12.25 & \\
\hline \multirow[t]{2}{*}{ Standing broad jump (cm) } & Female & 119.30 & 1.83 & 115.70 & 122.90 & $<0.001 \ddagger$ \\
\hline & Male & 135.42 & 2.08 & 131.33 & 139.51 & \\
\hline \multirow[t]{2}{*}{ Medicine-ball throw $(\mathrm{cm})$} & Female & 218.28 & 3.29 & 211.80 & 224.75 & $<0.001 \ddagger$ \\
\hline & Male & 248.91 & 3.74 & 241.56 & 256.27 & \\
\hline \multirow[t]{2}{*}{ Handgrip strength $\mathrm{R}(\mathrm{kg})$} & Female & 16.88 & 0.36 & 16.17 & 17.58 & $0.006 \ddagger$ \\
\hline & Male & 18.53 & 0.41 & 17.72 & 19.33 & \\
\hline \multirow[t]{2}{*}{ Handgrip strength $\mathrm{L}(\mathrm{kg})$} & Female & 15.54 & 0.35 & 14.86 & 16.22 & $0.003 \ddagger$ \\
\hline & Male & 17.27 & 0.39 & 16.50 & 18.04 & \\
\hline \multirow[t]{2}{*}{$\mathrm{M}-\mathrm{K}$ power stair test } & Female & 34.62 & 1.16 & 32.35 & 36.90 & $<0.001 \ddagger$ \\
\hline & Male & 43.88 & 1.31 & 41.29 & 46.46 & \\
\hline
\end{tabular}

*Bonferroni's test; $S E=$ standard error; $\mathrm{Cl}=$ confidence interval; $\mathrm{LB}=$ lower bound; $\mathrm{UB}=$ upper bound; MANCOVA = multivariate analysis of covariance.

$\dagger p \leq 0.05$;

$\pm p \leq 0.01$

reach the line on 2 consecutive occasions, they were stopped and the number of completed 20-m laps was recorded. The $20-\mathrm{m}$ multistage shuttle run has shown an intraclass correlation coefficient (ICC) of 0.98 .

Left and right sit-andreach: The subjects were seated with their legs joined and outstretched. The soles of their feet were supported on a standardized wooden box (Well Box). By means of a trunk inflection, the subjects were required to reach with the index finger (arms joined and hands superimposed) the maximum attainable distance as marked on the box. The participant was asked to stretch 4 times and hold the position for 1 second during the fourth attempt. The furthest distance the subject was able to reach was recorded in centimeters. The ICC of data for left and right sit-and-reach was 0.96 and 0.97, respectively.

Trunk lift: The subjects raised the torso as high as

(Margaria-Kalamen power stair test). Curl-ups, push-ups, 20-m multistage shuttle run, sit-and-reach, and trunk lift were evaluated using field tests from the FITNESSGRAM test battery (27). Standing broad jump, hand grip strength and flamingo balance were assessed using EUROFIT test battery (1). Shuttle run agility test was evaluated using the AAHPERD test battery (3). In the Margaria-Kalamen power stair test, the protocol was used as described by George et al. (13). Medicine-ball throw was evaluated using the protocol described by Mayhew et al. (26).

Twenty-m multistage shuttle run: This test involved continuous running between 2 lines $20 \mathrm{~m}$ apart in time to recorded beeps. The subjects ran between the 2 lines, turning when signaled by the recorded beeps. After about 1 minute, a sound indicated an increase in speed, and the beep rhythm accelerated. This pattern was continued at intervals of 1 minute per rhythm level. When the participants failed to possible from the floor from a prone position, while keeping the eyes on an object placed on the floor in line with the eyes. This position was held while the distance from the floor to the chin was measured. Two trials were given, and the furthest distance was measured in centimeters. The trunk lift has shown an ICC of 0.94 .

Twenty-m sprint running: In a track measuring $20 \mathrm{~m}$ in length, the subjects were required to cover the distance in the shortest time they could. Time to run $20 \mathrm{~m}$ was obtained using photocells (Brower Timing System, Fairlee, VT, USA). Three trials were performed, and the best time scored (seconds and hundredth) was registered. The sprint running (time) has shown an ICC of 0.96 .

Shuttle run agility test: The subjects were asked to tack and shift, at the fastest pace they were capable of, within an area delimited by 2 lines $(9.14 \mathrm{~m}$ apart each other). Two blocks were placed on the line opposite the starting line. On 
TABLE 3. Effect of the somatotype, physical activity, and body fat factors on the physical performance of boys and girls: MANCOVA.

\begin{tabular}{llcccc}
\hline \multicolumn{1}{c}{ Effect } & $F$ & $p$ & Partial eta squared & Observed power \\
\hline Male & Fat mass (\%) & 0.599 & 0.862 & 0.060 & 0.359 \\
& Physical activity & 2.217 & $0.010^{\star}$ & 0.190 & 0.960 \\
& Endomorphic & 1.196 & 0.286 & 0.113 & 0.705 \\
& Mesomorphic & 3.379 & $<0.001^{\star}$ & 0.264 & 0.998 \\
& Ectomorphic & 4.314 & $<0.001^{\star}$ & 0.314 & 1,000 \\
Female & Fat mass (\%) & 2.585 & $0.002^{\star}$ & 0.205 & 0.984 \\
& Physical activity & 1.554 & 0.100 & 0.135 & 0.843 \\
& Endomorphic & 3.444 & $<0.001^{\star}$ & 0.256 & 0.998 \\
& Mesomorphic & 2.131 & $0.013^{\dagger}$ & 0.176 & 0.952 \\
& Ectomorphic & 2.422 & $0.005^{\star}$ & 0.195 & 0.976 \\
\hline
\end{tabular}

${ }^{*} p \leq 0.01$

$\dagger p \leq 0.05$.

balance was lost. The number of such falls occurring over 60 seconds was then counted. The Flamingo balance has shown an ICC of 0.93 .

Standing long jump: The participants stood feet slightly apart (toes behind a starting line) and jumped as far forward as possible. Three trials were given, and the furthest distance was measured in centimeters from the starting line to the heel of the foot nearest to this line. The standing long jump has shown an ICC of 0.97 .

Curl-ups: The participants were required to bend their knees at approximately $140^{\circ}$,

a given signal, the participant sprinted to the opposite line, picked up a block of wood, ran back, and placed it on or beyond the starting line. Then, turning without pause, they ran back to retrieve the second block carrying it back across the finishing line. Three trials were performed, and the best time scored (seconds and hundredths) was registered. This test has shown an ICC of 0.98 .

Flamingo balance: Balancing on a preferred leg, the subject was required to flex the free leg at the knee with the foot of this leg held close to the buttocks. The stopwatch was stopped each time the participants lost balance (either by falling off the beam or letting go of the foot being held). The exercise was then recommenced and again timed until feet flat on the floor, arms straight and parallel to the trunk with palms of hands resting on the mat. The fingers were fully extended and the head placed in contact with the mat. One measuring strip was placed on the mat under the legs. The participant then curled up slowly, sliding the fingers across the measuring strip until the fingertips reached the other side, then curled back down until the head touched the mat. The number of correct curl-ups performed in a cadence of 20 curl-ups per minute ( 1 curl-up every 3 seconds) was then scored. This test has shown an ICC of 0.97 .

Push-ups: The participants were positioned with hands and toes touching the floor, body and legs in a straight line, feet slightly apart, arms at shoulder-width apart, extended and at right angles to the body. Keeping the back and knees straight, the subject then lowered the body to the point at which there was a $90^{\circ}$ angle at the elbows, with the upper arms parallel to the floor. The total number of correct pushups was recorded during a cadence of 20 complete push-ups per minute (1 complete push-up every 3 seconds). This test has shown an ICC of 0.97 .

Medicine-ball throw: The subjects were seated with the backside of the trunk in touch with a wall. They were required to hold a medicine ball (Bhalla International, Vinex Sports, Meerut, 
TABLE 5. Correlations between canonical coefficients and variables.

\begin{tabular}{|c|c|c|c|c|}
\hline & & \multicolumn{3}{|c|}{ Canonical dimensions ${ }^{*}$} \\
\hline & & 1 & 2 & 3 \\
\hline \multirow[t]{14}{*}{ Performance variables } & 20-m Shuttle run (a.u.) & 0.708 & -0.224 & 0.035 \\
\hline & 20-m Sprint (s) & -0.465 & 0.142 & -0.515 \\
\hline & 9.14-m Shuttle run $(\mathrm{s})$ & -0.491 & 0.049 & -0.507 \\
\hline & Flamingo balance (faults) & -0.262 & 0.373 & 0.114 \\
\hline & Sit-and-reach R $(\mathrm{cm})$ & 0.139 & -0.349 & -0.318 \\
\hline & Sit-and-reach L (cm) & -0.004 & -0.374 & -0.262 \\
\hline & Trunk lift $(\mathrm{cm})$ & -0.109 & -0.121 & -0.337 \\
\hline & Curl-ups (a.u.) & 0.494 & -0.010 & 0.024 \\
\hline & Push- ups (a.u.) & 0.274 & -0.026 & 0.031 \\
\hline & Standing broad jump $(\mathrm{cm})$ & 0.555 & -0.148 & 0.459 \\
\hline & Medicine-ball throw $(\mathrm{cm})$ & -0.034 & 0.333 & 0.332 \\
\hline & Handgrip strength $\mathrm{R}(\mathrm{kg})$ & -0.201 & 0.308 & 0.519 \\
\hline & Handgrip strength $L(\mathrm{~kg})$ & -0.202 & 0.303 & 0.412 \\
\hline & $\mathrm{M}-\mathrm{K}$ power stair test & 0.082 & 0.243 & 0.099 \\
\hline \multirow{5}{*}{$\begin{array}{l}\text { Eigenvalue and } \\
\text { variance }\end{array}$} & Eigenvalue & 1.417 & 0.780 & 0.377 \\
\hline & $\%$ of Variance & 52.2 & 28.7 & 13.9 \\
\hline & $\%$ of Cumulative variance & 52.2 & 80.9 & 94.8 \\
\hline & Canonical correlations & 0.766 & 0.662 & 0.523 \\
\hline & Wilks lambda & 0.147 & 0.355 & 0.632 \\
\hline \multirow{4}{*}{ Multivariate tests } & $F$ & 7.420 & 4.839 & 2.748 \\
\hline & DF 1 & 90.0 & 70.0 & 52.0 \\
\hline & DF 2 & 1643.1 & 1394.3 & 1136.9 \\
\hline & $p$ & $<0.001 \dagger$ & $<0.001 \dagger$ & $<0.001 \dagger$ \\
\hline
\end{tabular}

*Significant canonical dimensions; DF $1=$ hypothesis degrees of freedom; DF $2=$ error degrees of freedom.

$\dagger p<0.01$

step to the ninth step was then recorded. The test was repeated thrice and the fastest time recorded in hundredths of seconds. Power (kilograms per meter per second) was calculated as follows: Power $=$ body mass (kilograms) $\times$ vertical distance between steps (meters)/ time (seconds). The Margaria-Kalamen power stair has shown an ICC of 0.97 .

\section{Statistical Analyses}

Standard statistical methods were used for the calculation of the means and $S D$ s. Intraclass correlation coefficient was used to determine between-subject reliability of selected tests. Independent samples $t$-test was used to check gender differences in anthropometric and morphological parameters and physical activity indexes. For the analysis of statistical differences between boys and girls on physical performance varia-

India) weighing $2 \mathrm{~kg}$ (Vinex, model VMB-002R) with their hands (abreast of chest) and throw it forward over the maximum distance possible. Hip inflection was not allowed nor withdrawal of the trunk away from the wall. Three trials were given, and the furthest throw was measured in centimeters from the wall to the first point at which the ball made contact with floor. The medicine-ball throw has shown an ICC of 0.97 .

Left and right hand grip strength: This was measured by the Jamar $(0-200 \mathrm{lb})$ hydraulic hand dynamometer. Each participant stood erect in the $90^{\circ}$ elbow flexion position. The handle of the dynamometer was adjusted if required. The subjects were then instructed to exert maximal grip (for about 3 seconds), interrupted by brief pauses (of about 1 minute). No other body movement was allowed. Three trials were given for each hand separately and the best score recorded in kilograms was chosen for analysis. The ICC of data for left and right hand grip strength was 0.99 and 0.96 , respectively.

Margaria-Kalamen power stair: The participant was placed ready at the starting line $6 \mathrm{~m}$ in front of the first step. On command, the subject sprinted to and up the flight of steps, taking preferably 3 steps at a time in the attempt to mount the steps as fast as possible. The time taken to get from the third bles, a multivariate analysis of covariance (MANCOVA) was used, having as factors, in addition to sex, the maturation, and the fat mass, physical activity, endomorphic, mesomorphic and ectomorphic as covariates. The normality of the residuals of MANCOVA was checked by applying the KolmogorovSmirnov test and the homogeneity of variance-covariance matrix, was tested by the Box $M$ test $(M=435.60, F[315,36950.5]=$ $1.22, p \leq 0.05$ ). Because it was not verified, this assumption we used the Pillai's Trace test statistics. It should be noted that the correlations between the dependent variables ranged between 0.006 and 0.647 . When statistically significant differences were observed between boys and girls, an analysis of covariance (ANCOVA) was estimated for each dependent variable, followed by Bonferroni's post hoc comparison tests. By ANCOVA, it was also possible to analyze the statistical significance and effect size of gender in the physical performance variables. To determine which of the somatotype, physical activity, and body fat factor had a greater influence on the motor performance of both boys and girls, a MANCOVA was estimated for each gender, with the maturation as a factor and the fat mass, physical activity, and somatotype as covariates. The normality of the residuals was validated by the Kolmogorov-Smirnov univariate and the homogeneity of variance-covariance matrix was validated by the Box 


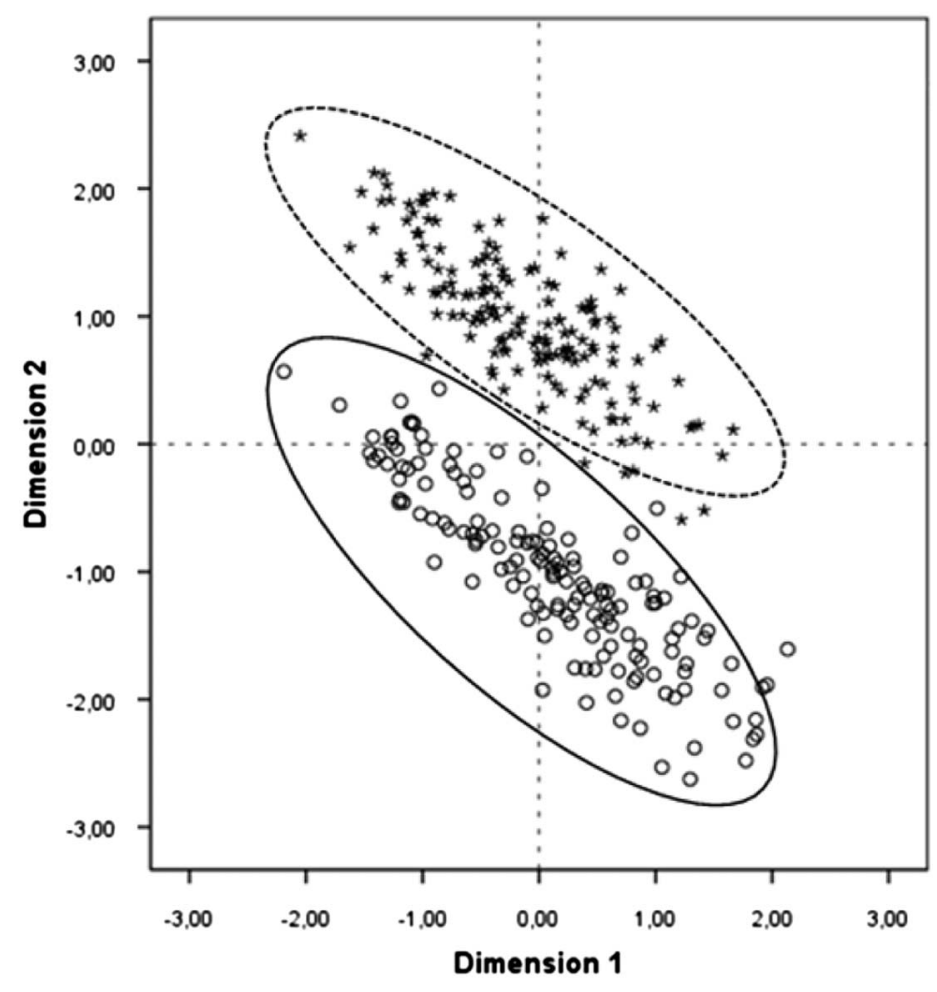

Figure 1. Scatterplot between dimension 1 and dimension 2 by sex.

observe that girls registered higher mean values of $\%$ FAT, ENDO, and ECTO. On the other hand, boys showed higher values in the MESO component and in all levels of physical activity (school, sport, leisure time, and total physical activity indexes). However, these differences were only significant in the MESO $(t=$ $-7.897, p \leq 0.001)$ and ECTO $(t=2.161, p \leq 0.05)$ components, and school $(t=-2.392$, $p \leq 0.05)$, sport $(t=-5.594$, $p \leq 0.001)$ and total $(t=$ $-4.330, p \leq 0.001)$ physical activity indexes. In \%FAT, ENDO and leisure-time index, gender differences were not statistically significant.

In the analysis of gender differences in physical performance, the MANCOVA showed that physical fitness variables had a Cronbach's Alpha of 0.705 , which indicates that they have a reasonable reliability for the physical performance measurement. We

M test (Girls: $M=190.47, F[105,4664.4]=1.12, p>0.05$; Boys: $M=385.41, F[210,5606.2]=1.14, p>0.05)$, so we used Wilk Lambda test. The reliability of physical performance was analyzed with Cronbach's alpha.

We also carried out a canonical correlation to understand which of the performance variables explained the data variability more. We first found how many dimensions were required to understand the existing associations. The tests of dimensionality indicated the existence of 3 canonical dimensions statistically significant. The first dimension had a canonical correlation of 0.766 , with an eigenvalue of 1.42 and an explained variance of $52.2 \%$. The second dimension showed a canonical correlation of 0.662 , representing an eigenvalue of 0.78 and an explained variance of $28.7 \%$. The third dimension had a canonical correlation of 0.523 , an eigenvalue of 0.377 and an explained variance of $13.9 \%$. Together $97.7 \%$ of variance was explained. Then, we calculated the correlation coefficients between the canonical dimensions and the physical performance variables, and the standardized scores for each dimension were represented graphically. Data were analyzed using SPSS 15.0. The statistical significance was set at $p \leq 0.05$.

\section{Results}

Regarding gender differences in anthropometric and morphological parameters and physical activity indexes, we can observed a significant and high-sized effect of gender in all physical performance measures (Pillai's Trace $=0.28, F[14$, $291]=8.13, p \leq 0.01, \eta_{\mathrm{p}}^{2}=0.28$, Power $\left.=1.00\right)$. However, we found that the effect of Tanner's stage was not significant (Pillai's Trace $=0.44, F[14,291]=0.97, p>0.05, \eta_{\mathrm{p}}^{2}=0.44$, Power $=0.61$ ). Through the ANCOVA, estimated for each dependent variable when statistically significant differences were observed between boys and girls, it was observed that the boys were superior to the girls on tests of aerobic capability, speed, agility, explosive strength, and maximum isometric strength. The girls were superior in flexibility of the lower back and hamstrings and balance (Table 2).

Regarding MANCOVA estimated for the girls, we observed a significant and high-sized effect of endormophic in all physical performance measures, followed by body fat, Ectomorphic and Mesomorphic, with significant but medium-sized effect. The physical activity did not have, for girls, a statistically significant effect on physical performance variables. In the boys, there was a significant and high-sized effect of Ectomorphic and Mesomorphic in all physical performance measures, followed by physical activity with significant but medium-sized effect. The variables body fat and endomorphic did not have, for boys, a statistically significant effect on physical performance variables (Table 3). 


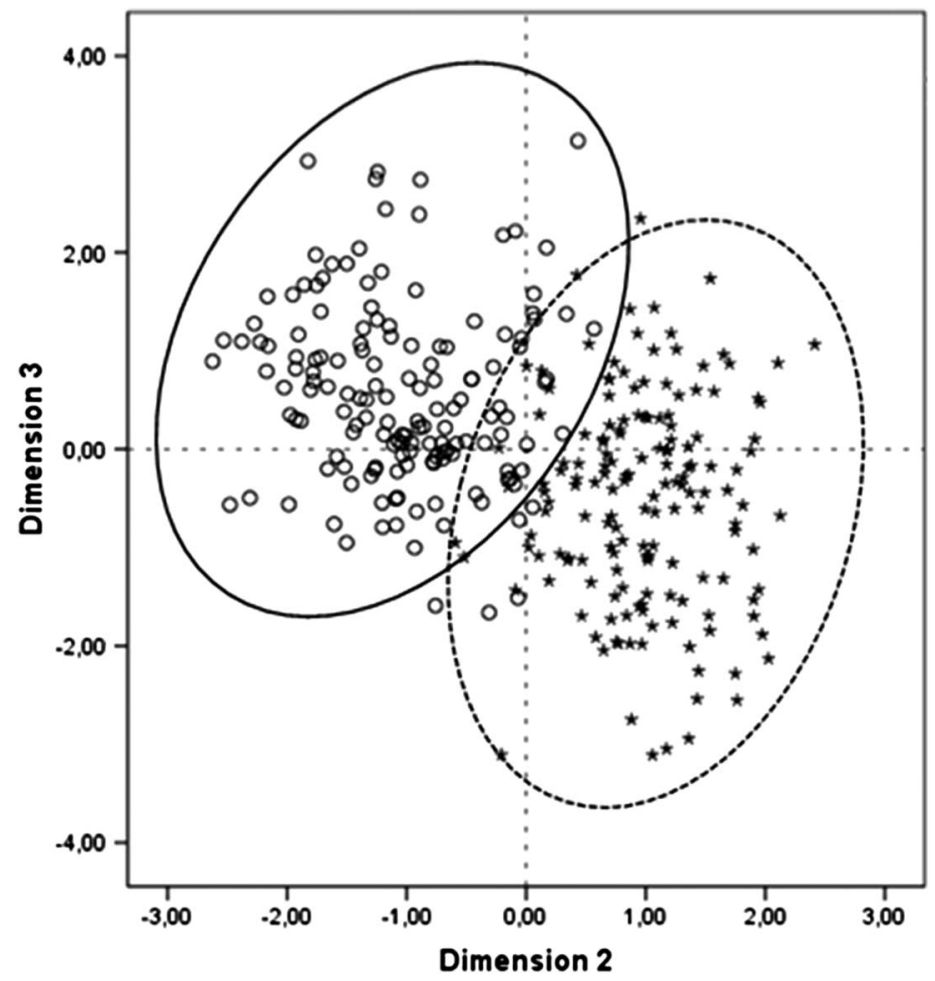

Figure 2. Scatterplot between dimension 2 and dimension 3 by sex.

Through the ANCOVA, we observed that gender differences in physical fitness were greater in the explosive strength of upper and lower limbs (medicine-ball throw and standing broad jump), although with a medium-size effect of gender. In the opposite direction, gender differences were smaller in the muscle strength and endurance (curl-ups and push-ups) and trunk extensor strength and flexibility (trunk lift), followed by speed (20-m run) and balance (flamingo balance) (Table 4).

Table 5 shows the correlation coefficients between the canonical dimensions and physical performance variables. The performance variables with the greatest effect on the first dimension were the endurance (0.708), explosive strength of lower limbs (0555), abdominal muscular endurance $(0.494)$, agility $(-0.491)$, and speed $(-0.465)$. In the second dimension, the variables flexibility of the lower back and hamstrings (right: -0.349 , and left: -0.374 ) and balance (0.373) were the ones that had a stronger correlation with this dimension. In the third dimension the speed $(-0.515)$ and agility $(-0.507)$ were the variables with the highest correlation with this dimension. (Table 5)

Figure 1 shows the standardized scores for the first dimension (it has the variables with the greatest positive effect the endurance, explosive strength of lower limbs and abdominal muscular endurance, in which boys have greater values than the average, and the greatest negative effect the speed and agility, where the boys have smaller values than the average), and the second dimension (it has the variable with the greatest positive effect the balance, where the boys have a number of faults greater than the average, and greatest negative effect the flexibility of the lower back and hamstrings, where the boys have smaller values than the average). We observed that the scores of the boys are more concentrated in the fourth quadrant (values above the average in the first dimension and below the average in the second dimension), whereas in the girls, the values are more concentrated in the first quadrant (values above the average in the second dimension and below the average in the first dimension).

Figure 2 shows the standardized scores for the second and third dimensions. The third dimension has the variables with the greatest positive effect the maximum isometric strength and explosive strength of lower limbs, in which the boys have greater values than the average, and the greatest negative effect the speed and agility, where the boys have smaller values than the average. It is observed that the scores of the boys are more concentrated in the second quadrant (values below the average in the second dimension, and above the average in the third dimension), whereas in the girls, the values are more concentrated in the fourth quadrant (values above the average in the second dimension and below the average in the third dimension).

\section{Discussion}

The purpose of this study was to analyze in which physical capabilities boys and girls are closer or divergent. An additional objective was to find which of the body fat, physical activity, and somatotype factor is more interactive with prepubescent children's physical fitness level. The main results suggested that the somatotype had the greatest influence on the motor performance of both boys and girls (ectomorphic and mesomorphic in the boys and endomorphic in the girls). The physical activity in the girls, and the endomorphic and body fat in the boys, had no significant 
effect. The difference between boys and girls in the physical fitness was greater in the explosive strength of upper and lower limbs, and smaller in the abdominal and upper limbs muscular endurance, and trunk extensor strength and flexibility, followed by speed and balance.

The girls studied showed higher values of \%FAT, ENDO, and ECTO compared with that shown by the boys. The higher percentage values of body fat in girls are consistent with the findings of several studies in the literature that report higher such values for women in general $(8,20,37)$. ENDO expresses the degree of adiposity development (23) being a variable close to \% FAT, either in terms of definition or the way they are both calculated so that values here also show higher for girls than for boys (23). This body fat represents an inert noncontributory load and thus an increased metabolic cost for children, making them less efficient in terms of cardiorespiratory response and performance on tests that require lifting and propulsion tasks $(4,11,39)$. The ECTO represents the relative thinness of the subject (23) and therefore associates negatively with strength (23). At the chronological ages under discussion, it seems that the weighting index, that is, the quotient of height by the cube root of body weight, which is based on the ECTO (23), is in favor of girls. This becomes more noticeable if we consider that the height growth curves in boys and girls intersect for a time, referred to as "crossing over," when girls overtake boys in stature, a stage that coincides with prepuberty (23). The boys showed higher values in the MESO component and in all levels of physical activity (school index, sport index, leisure-time index, and total index) relative to the girls. The main literature also refers to higher MESO values $(23,24)$ and higher levels of physical activity $(15,20,37)$ in male subjects. The MESO represents the relative skeletalmuscle magnitude (robustness) and therefore associates positively with strength and motor performance in general (23). Similarly, higher levels of physical activity are associated with a better physical fitness of children $(15,20,31,39)$.

The curiousity in this study is that the variables with no significant effect on the physical fitness of boys are the ones that had the greatest effect on the performance of girls (endomorphic and body fat). The greater robustness (23) and higher levels of physical activity (37) of the boys, including the higher levels of body fat $(8,20)$ and a more sedentary lifestyle (37) of the girls, seem to have influenced the effect of each of these factors on the physical fitness of boys and girls. No less interesting is that the primary components of the somatotype had the greatest influence on the motor performance of both boys and girls (ectomorphic and mesomorphic in the boys and endomorphic in the girls), and therefore, the relative skeletal-muscle magnitude of the boys and the degree of adiposity development of the girls seems to have reduced the dependence of the other factors on the motor performance. This fact highlights the importance of the morphological typology in the prepubescent children's physical fitness level and seems to suggest that one cannot neglect the limits imposed by what is a manifestation of genetic determinism, observed from the morphoconstitutional point of view, because the presence or absence of certain physical traits seems to be determinant on the physical performance of both boys and girls.

In terms of gender differences in motor performance, the results confirm those of other studies that report the superiority of boys in tests of aerobic fitness and muscular strength and of girls in tests of balance and flexibility $(8,15,20)$. Additionally, our findings indicate that the difference between boys and girls in physical fitness was greater in the explosive strength of upper and lower limbs, although with a medium-size effect of gender. In the opposite direction, gender differences were smaller in the muscle strength and endurance (curl-ups and push-ups) and trunk extensor strength and flexibility, followed by speed, and balance. Looking for the canonical correlation, the physical performance variables that most explain the variability of the data were the endurance, speed, agility, hand grip strength, abdominal muscular endurance, and explosive strength of lower limbs, in which boys are better, and flexibility of the lower back and hamstrings and balance, in which girls scored better. According to several studies in the literature, the boys are superior to girls in aerobic fitness because, among other factors such as higher levels of physical activity $(15,20,39)$ and lower fat mass $(4,11,30)$, to other factors mainly linked to the cardiac size and oxygen-carrying capacity (i.e., left ventricular inner diastolic diameter, maximal heart rate, and maximal stroke volume) (10). These factors seem to determine the difference found between boys and girls in cardiorespiratory fitness. In the same way, the fat-free mass or lean body mass statistically higher in boys and higher levels of physical activity permits a better muscular strength $(20,39)$. However, the absence of statistically significant differences between boys and girls in the muscular endurance tests (curl-ups and push-ups) corroborates the results of previous studies with children of similar age (7) and may be because the weight of the boys, close to the weight of girls in prepubertal stage (23), is an extra load to be moved during weight-bearing tasks, added to the fact that the boys still present a reduced muscle mass in prepubertal ages, because the effects of circulating androgens, particularly testosterone, only manifest themselves at puberty (23). Also, the lower values for body fat recorded by boys give them an advantage on tests of speed (11) and agility $(4,11)$, and the higher levels of physical activity permits better performance in the same capabilities $(31,36)$. Some approximation found in speed between boys and girls, may be because speed is a specific capability, highly dependent on the influence of genetic factors such as neuromuscular components and muscle fiber quality (i.e., fiber type proportion), and the high degree of gene transfer implied in this aptitude is today recognized (21). Regarding the flexibility, there are many reports that girls have larger range of motion. Some of the factors presented are the difference of 
extensibility of muscle and tendon tissues, the greater passive dorsiflexion angle of girls, because boys have a higher muscle volume, and dynamic property of tendon tissues (18). There are also studies in the literature referring to the absence of significant associations of this capability with physical activity levels (15), and skeletal-muscle magnitude (robustness), favorable to boys (34). There are even studies that report a negative association between the physical activity levels with performance on tests of flexibility (20). However, in this capability, the difference by gender there occupies a place emphasized during periods of rapid growth (28), which may explain in part the proximity between boys and girls in the trunk lift, associated to the fact that this capability is also very dependent of the upper body strength (27). The study of Monyeki et al. (28) showed that boys at this age can be more flexible than girls. Also in the balance boys and girls showed some proximity. These results may be obtained because in the prepubertal period the difference in stature between boys and girls gradually decreases, as a result of the growth velocity of girls at this stage that is higher than for boys, reaching the peak height velocity earlier (23). A greater stature leads to a high body's center of mass, in turn responsible for increased postural instability on balance exercises (2). However, other factors absent from this study may account for the approximation or divergence of boys and girls in physical fitness. Such factors may include, among others, different practice opportunities (38) or the preference for activities that require more endurance, strength, and speed or balance and flexibility (6).

\section{Practical Applications}

It has been observed that there is an apparent decrease in the interest of children in physical education classes and regular physical activity practice at school. This seems to be partly because of the lack of planning that takes into account the success of children in the execution of the exercises respecting the differences among students, including boys and girls. The knowledge of the magnitude of the differences between boys and girls in physical fitness (greater in the explosive strength of upper and lower limbs, and smaller in the abdominal and upper limbs muscular endurance and trunk extensor strength and flexibility, balance and speed), can help in the planning of activities that take into account the success of both boys and girls, and thus, increase levels of physical activity and physical fitness at school. However, the results seem to suggest that one cannot neglect the influence of genetic determinism, observed from the morpho-constitutional point of view, because the presence of certain physical traits (ectomorphic and mesomorphic in the boys and endomorphic in the girls) have shown to be determinant in prepubescent children's physical fitness level.

\section{ACKNOWLEDGMents}

The authors would like to thank all the children who participated in this research. They would also like to graciously thank the reviewers that took the time to critique this manuscript. The authors have no professional relationships with any companies or manufacturers identified in this study. The results of this study do not constitute endorsement of the product either by the authors or by the National Strength and Conditioning Association.

\section{REFERENCES}

1. Adam, C, Klissouras, V, Ravassolo, M, Renson, R, Tuxworth, W, Kemper, H, Van Mechelen, W, Hlobil, H, Beunen, G, LevarletJoye, H, and Van Lierde, A. Eurofit. Handbook for the Eurofit Test of Physical Fitness. Rome, Italy: Council of Europe. Committee for the Development of Sport, 1988.

2. Allard, $P$, Nault, $M$, Hinse, S, Leblawc, R, and Labelle, $H$. Relationship between morphologic somatotypes and standing posture equilibrium. Ann Hum Biol 28: 624-633, 2001.

3. American Alliance for Health, Physical Education, Recreation and Dance. Youth Fitness Test Manual. Washington, DC: AAHPERD, 1976.

4. Artero, EG, España-Romero, V, Ortega, FB, Jiménez-Pavón, D, Ruiz, JR, Vicente-Rodríguez, G, Bueno, M, Marcos, A, GómezMartínez, S, Urzanqui, A, González-Gross, M, Moreno, L, Gutiérrez, A, and Castillo, MJ. Health-related fitness in adolescents: underweight, and not only overweight, as an influencing factor. The AVENA study. Scand J Med Sci Sport 20: 418-427, 2010.

5. Baecke, JA, Burema, J, and Frijiters, JE. A short questionnaire for the measurement of habitual physical activity in epidemiological studies. Am J Clin Nutr 36: 936-942, 1982.

6. Branta, C, Haubenstricker, J, and Seefeldt, V. Age changes in motor skills during childhood and adolescence. Exercise Sport Sci Rev 12: 467-520, 1984.

7. Castro-Piñero, J, González-Montesinos, JL, Mora, J, Keating, XD, Girela-Rejón, MJ, Sjöström, M, and Ruiz, JR. Percentile values for muscular strength field tests in children aged 6 to 17 years: Influence of weight status. J Strength Cond Res 23: 2295-2310, 2009.

8. Cepero, MR, López, R, Suárez-Llorca, C, Andreu-cabrera, E, and Rojas, FJ. Fitness test profiles in children aged 8-12 years old in Granada (Spain). J Hum Sport Exerc 6: 135-146, 2011.

9. Coleman, KJ, Heath, EM, and Alcala, IS. Overweight and aerobic fitness in children in the United States/Mexico border region. Rev Panam Salud Públ/Pan Am J Public Health 15: 262-271, 2004.

10. Dencker, M, Thorsson, O, Karlsson, M, Lindén, C, Eiberg, S, Wollmer, P, and Andersen, B. Gender differences and determinants of aerobic fitness in children aged 8-11 years. Eur J Appl Physiol 99: 19-26, 2007.

11. Dumith, SC, Ramires, VV, Souza, MA, Moraes, DS, Petry, FG, Oliveira, ES, Ramires, SV, and Hallal, PC. Overweight/obesity and physical fitness among children and adolescents. J Phys Act Health 7: 641-648, 2010.

12. Faigenbaum, AD, Westcott, WL, Micheli, LJ, Outerbridge, AR, Long, CJ, LaRosa-Loud, R, and Zaichkowsky, LD. The effects of strength training and detraining on children. J Strength Cond Res 10: 109-114, 1996.

13. George, JD, Fisher, AG, and Vehrs, PR. Laboratory Experiences in Exercise Science. Boston, MA: Jones \& Bartlett Publishers, Inc, 1994.

14. Haff, GG. Roundtable discussion: Youth resistance training. Strength Cond J 25: 49-64, 2003.

15. Hands, B, Larkin, D, Parker, H, Straker, L, and Perry, M. The relationship among physical activity, motor competence and healthrelated fitness in 14-year-old adolescents. Scand J Med Sci Sports 19: 655-663, 2009.

16. Heath, $\mathrm{BH}$ and Carter, JE. Growth and somatotype patterns of Manus children, territory of Papua and New Guinea: Application of a modified somatotype method to the study of growth patterns. $\mathrm{Am}$ J Phys Anthropol 35: 49-67, 1971. 
17. Jakšić, D and Cvetković, M. Neural network analysis of somatotype differences among males related to the manifestation of motor abilities. Acta Kinesiologica 3: 107-113, 2009.

18. Kato, E, Oda, T, Chino, K, Kurihara, T, Nagayoshi, T, Fukunaga, T, and Kawakami, Y. Musculotendinous factors influencing difference in ankle joint flexibility between women and men. Int J Sport Health Sci 3: 218-225, 2005.

19. Kvaavik, E, Klepp, KI, Tell, GS, Meyer, HE, and Batty, GD. Physical fitness and physical activity at age 13 years as predictors of cardiovascular disease risk factors at ages 15, 25, 33, and 40 years: Extended follow-up of the Oslo Youth Study. Pediatrics 123: e80e86, 2009.

20. Lennox, A, Pienaar, AE, and Wilders, C. Physical fitness and the physical activity status of 15 -year-old adolescents in a semi-urban community. S Afr J Res Sport Phys Educ Recreation 30: 59-73, 2008.

21. Little, T and Williams, AG. Specificity of acceleration, maximum speed, and agility in professional soccer players. J Strength Cond Res 19:76-78, 2005.

22. Lyu, M and Gill, D. Perceived physical competence, enjoyment and effort in same-sex and coeducational physical education classes. $J$ Educ Psychol 31: 247-260, 2011

23. Malina, RM and Bouchard, C. Growth Maturation and Physical Activity. Champaign, IL: Human Kinetics, 1991.

24. Malina, RM, Bouchard, C, and Bar-Or, O. Grozeth, Maturation and Physical Activity. Champaign, IL: Human Kinetics, 2004.

25. Marfell-Jones, M, Olds, T, Stewart, A, and Carter, L. International Standards for Anthropometric Assessment. Potchefstroom, South Africa: ISAK, 2006.

26. Mayhew, JL, Ware, JS, Johns, RA, and Bemben, MG. Changes in upper body power following heavy-resistance strength training in college men. Int J Sports Med 18: 516-520, 1997.

27. Meredith, MD and Welk, GJ. Fitnessgram/Activitygram Test Administration Manual (4th ed.). Champaign, IL: Human Kinetics, 2007.

28. Monyeki, MA, Koppes, LJ, Kemper, HG, Monyeki, KD, Toriola, AL Pienaar, AE, and Twisk, JW. Body composition and physical fitness of undernourished South African rural primary school children. Eur J Clin Nutr 59:877-883, 2005.
29. Physical activity and cardiovascular health. NIH Consensus Development Panel on Physical Activity and Cardiovascular Health. J Am Med Assoc 276: 241-246, 1996.

30. Ortega, FB, Tresaco, B, Ruiz, JR, Moreno, LA, Matillas, M, Mesa, JL, Warnberg, J, Bueno, M, Tercedor, P, Gutiérrez, A, and Castillo, MJ. Cardiorespiratory fitness and sedentary activities are associated with adiposity in adolescents. Obesity 15: 1589-1599, 2007.

31. Reed, JA and Metzker, A. Relationships between physical activity and motor skills in middle school children. Percept Motor Skill 99: 483-494, 2004.

32. Reis, VM, Machado, JV, Fortes, MS, Fernandes, PR, Silva, AJ, Dantas, PS, and Filho, JF. Evidence for higher heritability of somatotype compared to body mass index in female twins. J Physiol Anthropol 26: 9-14, 2007.

33. Roetert, EP. The lack of childhood activity in the United States. Strength Cond J 26: 22-23, 2004.

34. Shukla, M, Venugopal, R, and Mitra, M. A cross sectional study of body composition somatotype and motor quality of rural and urban boys of Chhattisgarh. Int J Fitness 5: 1-7, 2009.

35. Slaughter, MH, Lohman, TG, Boileau, RA, Horswill, CA, Stillman, RJ, VanLoan, MD, and Bemben, DA. Skinfold equations for estimation of body fatness in children and youth. Hum Biol 60: 709-723, 1988

36. Sola, K, Brekke, N, and Brekke, M. An activity-based intervention for obese and physically inactive children organized in primary care: Feasibility and impact on fitness and BMI a one-year follow-up study. Scand J Prim Health 28: 199204, 2010.

37. Sveinsson, T, Arngrimsson, SA, and Johannsson, E. Association between aerobic fitness, body composition, and physical activity in 9- and 15-year-olds. Eur J Sport Sci 9: 141-150, 2009.

38. Thomas, JR and French, KE. Gender differences across age in motor performance: A meta-analyses. Psychol Bull 98: 260-282, 1985.

39. Tovar, G, Poveda, JG, Pinilla, MI, and Lobelo, F. Relationship between overweight, physical activity and physical fitness in schoolaged boys in Bogotá Colombia. Arch Latinoam Nutr 58: 265-273, 2008 . 